\title{
Phase transition for the dilute clock model
}

\author{
Inés Armendáriz; Pablo A. Ferrari† Nahuel Soprano-Loto
}

September 2, 2015

\begin{abstract}
We prove that phase transition occurs in the dilute ferromagnetic nearest-neighbour $q$-state clock model in $\mathbb{Z}^{d}$, for every $q \geq 2$ and $d \geq 2$. This follows from the fact that the Edwards-Sokal random-cluster representation of the clock model stochastically dominates a supercritical Bernoulli bond percolation probability, a technique that has been applied to show phase transition for the low-temperature Potts model. The domination involves a combinatorial lemma which is one of the main points of this article.
\end{abstract}

AMS 2000 Mathematics Subject Classification: 82B

Keywords: Dilute clock model, phase transition.

\section{Introduction}

The $q$-state clock model assigns a random spin to each site of $\mathbb{Z}^{d}$. The spins take values in a discrete set $S$ of equidistant angles or hours, hence the name. Let $\sigma=\left(\sigma_{x}, x \in \mathbb{Z}^{d}\right)$ be a spin configuration, $\sigma_{x}$ the angle of the spin at $x \in \mathbb{Z}^{d}$. Let $\mathcal{E}\left(\mathbb{Z}^{d}\right):=\{\langle x y\rangle:\|x-y\|=1\}$ be the set of edges connecting nearest neighbour sites, $\|\cdot\|$ the Euclidean norm. We study the dilute clock model associated to a disorder, namely a collection

$$
J=\left(J_{\langle x y\rangle}:\langle x y\rangle \in \mathcal{E}\left(\mathbb{Z}^{d}\right)\right)
$$

of independent identically distributed Bernoulli random variables with parameter $p$. A disorder realization $J$ and a finite set $\Lambda \subset \mathbb{Z}^{d}$ determine the Hamiltonian on spin configurations:

$$
H_{\Lambda, J}(\sigma):=\sum_{\substack{\langle x y\rangle \in \mathcal{E}\left(\mathbb{Z}^{d}\right) \\\{x, y\} \cap \Lambda \neq \emptyset}} J_{\langle x y\rangle}\left(1-\cos \left(\sigma_{x}-\sigma_{y}\right)\right) .
$$

When $q=2$, we recover the Ising model; as $q \rightarrow \infty$, the clock model approximates the $X Y$ model, which has a continuum of spin angles.

Given a set $\Lambda \subset \mathbb{Z}^{d}$ and configurations $\sigma, \eta \in S^{\mathbb{Z}^{d}}$, we write

$$
\sigma \stackrel{\Lambda}{=} \eta \quad \text { if } \quad \sigma_{x}=\eta_{x} \forall x \in \Lambda
$$

*Departamento de Matemática, UBA, Buenos Aires, Argentina. Email: iarmend@dm.uba.ar

${ }^{\dagger}$ Departamento de Matemática, UBA and IMAS-CONICET, Buenos Aires, Argentina, and IME-USP, São Paulo, Brazil. Email:pferrari@dm.uba.ar

${ }^{\ddagger}$ IMAS-CONICET, Buenos Aires, Argentina. Email: nsloto@dm.uba.ar 
The configuration $\eta$ plays the role of a boundary condition. The specification $\mu_{\Lambda, J}^{\eta}$ associated to a finite set $\Lambda$, a disorder $J$, and a boundary condition $\eta$ is the probability

$$
\mu_{\Lambda, J}^{\eta}(\sigma):=\frac{1}{Z_{\Lambda, J}^{\eta}} e^{-\beta H_{\Lambda, J}(\sigma)} \mathbf{1}\left[\sigma \stackrel{\Lambda^{c}}{=} \eta\right]
$$

where $\beta>0$ is a parameter proportional to the inverse temperature, $Z_{\Lambda, J}^{\eta}$ is the normalizing constant and $\Lambda^{c}=\mathbb{Z}^{d} \backslash \Lambda$. A Gibbs measure associated to the disorder $J$ is a probability $\mu_{J}$ that satisfies the DLR condition:

$$
\mu_{J} f=\int_{S^{\mathbb{Z}^{d}}} \mu_{J}(d \eta) \mu_{\Lambda, J}^{\eta} f
$$

for every finite subset $\Lambda \subset \mathbb{Z}^{d}$ and every local function $f: S^{\mathbb{Z}^{d}} \rightarrow \mathbb{R}$. Here, $\mu f$ denotes the expectation of $f$ with respect to $\mu$. The underlying $\sigma$-algebra where the Gibbs measures and the specifications are defined is the one generated by projections over finite subsets of $\mathbb{Z}^{d}$. We call $\mathcal{G}_{J}$ the set of Gibbs measures associates to $J$. Since $S$ is finite, $\mathcal{G}_{J}$ is not empty. In case $\left|\mathcal{G}_{J}\right|>1$, we say that phase co-existence occurs.

The homogeneous version of the model is obtained by taking $p=1$ or, equivalently, $J_{\langle x y\rangle} \equiv 1$ for every $\langle x y\rangle$. In this case, non-uniqueness methods such as the Pirogov-Sinai theory [PS75] or reflection positivity as in Frölich, Israel, Lieb and Simon [FILS78], see also Biskup [Bis09], prove that, for sufficiently low temperature, there exist at least $q$ different Gibbs measures. On the other hand, when the temperature is large enough, techniques similar to those developed by Dobrushin [Dob68] or by van den Berg and Maes [vdBM94] show that there exists only one Gibbs measure. Phase transition occurs when a system undergoes a change in its phase diagram depending on the value of a parameter; these results hence establish occurrence of phase transition for the homogeneous clock model.

Both Pirogov-Sinai theory and reflection positivity depend on the graph determined by the interacting edges in the Hamiltonian (2) being symmetric, an assumption that breaks down for the properly dilute model $p<1$. Instead, our main tool is the Fortuin-Kasteleyn randomcluster representation [KF69], originally introduced for the Ising and the Ashkin-Teller-Potts models, and then generalized to arbitrary models by Edwards and Sokal [ES88]; here, we build the clock model random-cluster representation in detail. The core idea of this approach is to relate non-uniqueness of Gibbs measure in the statistical-mechanical model to the existence of an infinite cluster in the random-cluster model: a percolation problem. It was first applied by Aizenman, Chayes, Chayes and Newman to study the phase diagram of the dilute Ising and Potts models in [ACCN87]; we presently adapt their ideas to our context.

Precisely, we derive a lower bound for the critical temperature: for every dimension $d$ and every number $q$ of spins, we take $p$ sufficiently large to guarantee that the disorder almost surely contains an infinite bond-percolation cluster, and then determine a value $\beta_{0}=\beta_{0}(q, d, p)>0$ such that there is more than one Gibbs measure at inverse temperatures $\beta>\beta_{0}$, for almost all disorders $J$. A crucial step in the proof consists of dominating the random-cluster probability associated to the clock model by a supercritical Bernoulli product probability on the bonds. While for the Potts model this domination is immediate, the clock model requires a combinatorial argument, given in Lemma 2.4.

The following is our main result.

Theorem 1.1. Let $p>p_{c}$, where $p_{c}$ is the Bernoulli bond percolation critical probability in $\mathbb{Z}^{d}$. Let the disorder $J$ be distributed as a product $P_{p}$ of i.i.d. Bernoulli random variables with parameter $p$. Then there exists $\beta_{0}>0$ such that, for $\beta>\beta_{0}$, the q-state dilute clock model associated to the random specifications $\mu_{\Lambda, J}^{\eta}$ defined in (4) exhibits phase co-existence for $P_{p}$-almost every realization of $J$. More precisely, $\beta>\beta_{0}$ implies $P_{p}\left(J:\left|\mathcal{G}_{J}\right| \geq q\right)=1$. 
The value $\beta_{0}$ in the later theorem depends on $d, q$ and $p$. We show in the Appendix that, for fixed $d$ and $p, \beta_{0}(q, d, p) \sim q^{2} \log q$ as $q \rightarrow \infty$, the same asymptotics provided by PigorovSinai theory and reflection positivity in the 2-dimensional homogeneous case. In particular, $\lim _{q \rightarrow \infty} \beta_{0}(q, d, p)=\infty$, implying that our approach is not suitable to study the $X Y$ model; see van Enter, Külske and Opoku [vEKO11] for results concerning the approximation of the $X Y$ model via the clock model. On the other hand, for $d \geq 3$ and $p=1$, reflection positivity computes a threshold $\beta_{0}$ independent of $q$, see Maes and Shlosman [MS11] for a discussion.

The ideas presented in this article can be further developed in two directions, which are explored by Soprano-Loto in collaboration with Roberto Fernández in a separate article [FSL]. The first one is a generalization of the current work to the so called Abelian spin models; see Dubédat [Dub11] for a precise definition. The second direction of research seeks to obtain a uniqueness criterion, also via random-cluster representation, at a higher level of generality.

Organization of the article. We introduce the random-cluster model and state the results leading to the proof of Theorem 1.1 in Section 2. Section 3 contains the proofs and the Appendix collects some auxiliary computations.

\section{Clock model and random-cluster in a finite graph}

We define the clock model and its random-cluster representation for a fixed non-oriented finite graph $(V, \mathcal{E})$ without loops or multiple edges, and not necessarily connected. We fix a non-empty subset $U \subset V$ playing the role of boundary. For simplicity, we suppose there are no edges connecting vertices in $U:\{\langle x y\rangle \in \mathcal{E}:\{x, y\} \subset U\}=\emptyset$.

In the case of the dilute clock in a finite set $\Lambda \subset \mathbb{Z}^{d}$, the boundary is given by $\partial \Lambda:=\{y \in$ $\left.\mathbb{Z}^{d} \backslash \Lambda, \exists x \in \Lambda:\|x-y\|^{2}=1\right\}$, and the vertex and edge sets are

$$
\Lambda \cup \partial \Lambda \quad \text { and } \quad\left\{\langle x y\rangle,\{x, y\} \not \subset \Lambda^{c},\|x-y\|=1, J_{\langle x y\rangle}=1\right\} .
$$

The clock model. Let $S$ be the set of angles defined by

$$
S:=\left\{\frac{2 \pi i}{q}: i=0, \ldots, q-1\right\} .
$$

Elements of $S$ are called spins and denoted $a, b$ and $c$, while spin or vertex-configurations in $S^{V}$ are denoted by $\sigma$ and $\eta$.

The clock Hamiltonian $H=H(V, \mathcal{E})$ is the function $H: S^{V} \rightarrow \mathbb{R}$ defined by

$$
H(\sigma):=\sum_{\langle x y\rangle \in \mathcal{E}}\left(1-\cos \left(\sigma_{x}-\sigma_{y}\right)\right) .
$$

We write $\sigma \stackrel{U}{=} a$ when $\sigma_{x}=a$ for all $x \in U$. The clock probability $\mu=\mu(V, U, \mathcal{E}, \beta)$ with 0-boundary condition is defined as

$$
\mu(\sigma):=\frac{1}{Z} e^{-\beta H(\sigma)} \mathbf{1}[\sigma \stackrel{U}{=} 0]
$$

where $\beta$ is a strictly positive parameter and $Z=Z(V, U, \mathcal{E}, \beta)$ is the normalizing constant.

The random-cluster measure. Define a weight function $W: S \rightarrow(0,1]$ by

$$
W(a):=e^{-\beta(1-\cos a)}
$$


and let $\mathcal{I}:=\{W(a), a \in S\}$ be its image. This set has cardinality $|\mathcal{I}|=k+1$, where $k=q / 2$ for even $q$ and $k=(q-1) / 2$ for odd $q$. Write $\mathcal{I}=\left\{t_{0}, t_{1}, \ldots, t_{k}\right\}$ with $0<t_{0}<t_{1}<\cdots<t_{k}=$ $W(0)=e^{-\beta(1-\cos 0)}=1$, and denote

$$
r_{0}:=t_{0}, \quad r_{i}:=t_{i}-t_{i-1}, \quad 1 \leq i \leq k .
$$

By construction $0 \leq r_{i} \leq 1$ for all $0 \leq i \leq k$, and $\sum_{i} r_{i}=1$.

Let $\theta$ be the probability on $\mathcal{I}$ given by

$$
\theta\left(t_{i}\right):=r_{i}, \quad 0 \leq i \leq k,
$$

and let $\hat{\phi}=\hat{\phi}(\mathcal{E}, \beta)$ be the product measure on the set of edge-configurations $\omega \in \mathcal{I}^{\mathcal{E}}$ with marginals $\theta$ :

$$
\hat{\phi}(\omega):=\prod_{\langle x y\rangle \in \mathcal{E}} \theta\left(\omega_{\langle x y\rangle}\right) .
$$

We say that an edge-configuration $\omega \in \mathcal{I}^{\mathcal{E}}$ and a vertex-configuration $\sigma \in S^{V}$ are compatible, and write $\omega \preceq \sigma$, if the value of $\omega$ on any edge is dominated by the weight of the gradient of $\sigma$ over that edge:

$$
\omega \preceq \sigma \quad \Leftrightarrow \quad \omega_{\langle x y\rangle} \leq W\left(\sigma_{x}-\sigma_{y}\right) \text { for every }\langle x y\rangle \in \mathcal{E} .
$$

Notice that if $\omega \preceq \sigma$ and $\omega_{\langle x y\rangle}=1$, then $\sigma_{x}=\sigma_{y}$; on the other hand, $\omega_{\langle x y\rangle}=0$ imposes no restriction on the values of $\sigma_{x}$ and $\sigma_{y}$.

We define the random-cluster probability $\phi=\phi(V, U, \mathcal{E}, \beta)$ on $\mathcal{I}^{\mathcal{E}}$ as the measure obtained from $\hat{\phi}$ by assigning to each edge-configuration $\omega$ a weight proportional to the number of vertexconfigurations $\sigma$ that are compatible with $\omega$ and satisfy the boundary condition, using $\hat{\phi}$ as reference measure:

$$
\phi(\omega):=\frac{1}{Z}|\{\sigma: \sigma \succeq \omega, \sigma \stackrel{U}{=} 0\}| \hat{\phi}(\omega) .
$$

Here $Z$ is the same normalizing constant appearing in (9).

The Edwards-Sokal coupling. Let $\hat{\mu}=\hat{\mu}(V, U)$ be the uniform probability on the set of vertex configurations $S^{V}$ that are identically 0 at sites in $U$ :

$$
\hat{\mu}(\sigma):=\frac{1}{q^{|V \backslash U|}} \mathbf{1}[\sigma \stackrel{U}{=} 0] .
$$

We define a joint edge-vertex probability $Q=Q(V, U, \mathcal{E}, \beta)$ on $\mathcal{I}^{\mathcal{E}} \times S^{V}$ by

$$
Q(\omega, \sigma):=\frac{1}{Z^{\prime}} \mathbf{1}[\omega \preceq \sigma] \hat{\phi}(\omega) \hat{\mu}(\sigma),
$$

where $Z^{\prime}:=Z / q^{|V \backslash U|}$ with $Z$ as in (9). That is, $Q$ is the product probability $\hat{\phi} \times \hat{\mu}$ conditioned to the compatibility event $\{(\omega, \sigma): \omega \preceq \sigma\} \subset \mathcal{I}^{\mathcal{E}} \times S^{V}$.

Theorem 2.1. Edwards-Sokal [ES88].

The measures $\phi$ and $\mu$ are respectively the first and second marginals of $Q$. 
We prove this theorem in Section 3. The measure $Q$ can be seen as a coupling between the clock measure $\mu$ and the random-cluster measure $\phi$. As a corollary, it follows that the conditional distribution under $Q$ of $\sigma$ given $\omega$ is uniform on the set of configurations compatible with $\omega$ and such that $\sigma \stackrel{U}{=} 0$ :

$$
Q(\sigma \mid \omega)=\frac{Q(\omega, \sigma)}{\sum_{\sigma^{\prime}} Q\left(\omega, \sigma^{\prime}\right)}=\frac{\hat{\mu}(\sigma) \mathbf{1}[\omega \preceq \sigma]}{\hat{\mu}\left(\sigma^{\prime}: \omega \preceq \sigma^{\prime}\right)} .
$$

This implies that a random vertex-configuration distributed according to $\mu$ may be sampled by first choosing an edge-configuration $\omega$ with law $\phi$, and then sampling a vertex-configuration uniformly among those that are compatible with $\omega$ and satisfy the boundary restriction. That is,

$$
\mu(\sigma)=\sum_{\omega \in \mathcal{I}^{\mathcal{E}}} \frac{\hat{\mu}(\sigma) \mathbf{1}[\omega \preceq \sigma]}{\hat{\mu}\left(\sigma^{\prime}: \omega \preceq \sigma^{\prime}\right)} \phi(\omega) .
$$

Given $x, y \in V$ and $\omega \in \mathcal{I}^{\mathcal{E}}$, we denote $x \stackrel{\omega}{\longleftrightarrow} y$ if there is a path of vertices $x_{1}, \ldots, x_{n} \in V$ with $x_{1}=x, x_{n}=y,\left\langle x_{i} x_{i+1}\right\rangle \in \mathcal{E}$ and $\omega_{\left\langle x_{i} x_{i+1}\right\rangle}=1$ for $1 \leq i \leq n-1$. We say that $x$ is connected to $U$ by an $\omega$-open path, and write $x \stackrel{\omega}{\longleftrightarrow} U$, when $x \stackrel{\omega}{\longleftrightarrow} y$ for some $y \in U$; let $x \stackrel{\omega}{\longleftrightarrow} U$ denote the complementary event. The $\mu$-marginal of the spin at $x$ can be related to the connection probabilities between $x$ and the boundary, under $\phi$ and $Q$ :

$$
\mu\left(\sigma: \sigma_{x}=a\right)=\phi(\omega: x \stackrel{\omega}{\longleftrightarrow} U) \mathbf{1}[a=0]+Q\left((\omega, \sigma): \sigma_{x}=a, x \stackrel{\omega}{\longleftrightarrow} U\right) .
$$

Identity (20) follows immediately from the coupling of Theorem 2.1 and the inclusion $\{(\omega, \sigma)$ : $x \stackrel{\omega}{\longleftrightarrow} U\} \subset\left\{(\omega, \sigma): \sigma_{x}=0\right\}$.

The coupling of Theorem 2.1 also implies that the $\mu$-probability of seeing a 0 at any site $x$ is larger than the probability of seeing any other spin plus the $\phi$-probability that $x$ be connected to the boundary. This is the content of the next result; its proof depends crucially on the combinatorial Lemma 2.4 stated later.

Proposition 2.2. Positive correlations.

For any vertex $x \in V$ and any spin $a \neq 0$,

$$
\mu\left(\sigma: \sigma_{x}=0\right) \geq \mu\left(\sigma: \sigma_{x}=a\right)+\phi(\omega: x \stackrel{\omega}{\longleftrightarrow} U) .
$$

Stochastic domination. Given $I \subset \mathbb{R}$, consider the partial order on $I^{\mathcal{E}}$ defined by $\omega \leq \omega^{\prime}$ if and only if $\omega_{\langle x y\rangle} \leq \omega_{\langle x y\rangle}^{\prime}$ for every $\langle x y\rangle \in \mathcal{E}$. A function $f: I^{\mathcal{E}} \rightarrow \mathbb{R}$ is said to be increasing if $f(\omega) \leq f\left(\omega^{\prime}\right)$ whenever $\omega \leq \omega^{\prime}$, while an event $E \subset I^{\mathcal{E}}$ is said to be increasing when its indicator function $f(\omega)=\mathbf{1}[\omega \in E]$ is. Given two probabilities $P$ and $P^{\prime}$ on $I^{\mathcal{E}}$, we say that $P$ is stochastically dominated by $P^{\prime}$, and write $P \leq_{s t} P^{\prime}$, if and only if $P f \leq P^{\prime} f$ for every increasing $f: I^{\mathcal{E}} \rightarrow \mathbb{R}$. This is equivalent to $P(E) \leq P^{\prime}(E)$ for any increasing event $E$.

Given $\rho \in[0,1]$, let $B_{\rho}$ be the Bernoulli product measure on $\{0,1\}^{\mathcal{E}}$ with parameter $\rho$. In order to stochastically compare $\phi$ and $B_{\rho}$ we consider them defined on the common space $I^{\mathcal{E}}$, where $I=\{0\} \cup \mathcal{I}$.

Theorem 2.3. Stochastic domination.

For any $\rho \in[0,1)$ there exists $\beta_{0}=\beta_{0}(\rho)>0$, independent of the graph $(V, \mathcal{E})$ and the boundary $U$, such that, if $\beta \geq \beta_{0}, B_{\rho}$ is stochastically dominated by $\phi$. 
The key to the proofs of Proposition 2.2 and Theorem 2.3 is the following combinatorial lemma, proved in Section 3.

Lemma 2.4. For every $x \in V, a \in S$ and $\omega \in \mathcal{I}^{\mathcal{E}}$,

$$
\left|\left\{\sigma: \sigma \preceq \omega, \sigma \stackrel{U}{=} 0, \sigma_{x}=a\right\}\right| \leq\left|\left\{\sigma: \sigma \preceq \omega, \sigma \stackrel{U}{=} 0, \sigma_{x}=0\right\}\right| .
$$

Equivalently,

$$
\hat{\mu}\left(\sigma: \sigma_{x}=a, \sigma \preceq \omega\right) \leq \hat{\mu}\left(\sigma: \sigma_{x}=0, \sigma \preceq \omega\right) .
$$

The lemma in fact holds for any spin set $S^{\prime}$ and weight function $W^{\prime}$ provided they satisfy certain symmetry properties: for any pair of elements $a, b \in S^{\prime}$ it must be possible to define a reflection $R=R_{a, b}: S^{\prime} \rightarrow S^{\prime}, R(a)=b$, such that $\left.i\right)$ it splits $S^{\prime}$ into two hemispheres $\operatorname{Hem}(a)$ and $\operatorname{Hem}(b), a \in \operatorname{Hem}(a), b \in \operatorname{Hem}(b)$, in such a way that $W^{\prime}(c-R(d))<W^{\prime}(c-d)$ implies $c$ and $d$ belong to the same hemisphere, and $i i) R$ preserves the compatibility of neighbouring vertices when applied to both spins. These extensions are explored in detail in [FSL].

In the dilute Potts model with $q$ spins, the Hamiltonian is given by $\sum_{\langle x y\rangle} J_{\langle x y\rangle} \mathbf{1}\left[\sigma_{x} \neq \sigma_{y}\right]$, and the associated random-cluster probability is defined on $\{0,1\}^{\mathcal{E}}$; see [GHM01, Gri06], for example. In this case, if $\sigma$ and $\omega$ are compatible, the values of $\sigma_{x}$ and $\sigma_{y}$ must coincide whenever $\omega_{\langle x y\rangle}=1$, and there are no restrictions if $\omega_{\langle x y\rangle}=0$. Call a connected component of the graph $\left(V,\left\{\langle x y\rangle: \omega_{\langle x y\rangle}=1\right\}\right)$ an $\omega$-cluster. Then $\omega \preceq \sigma$ implies that $\sigma$ is constant over each of the $\omega$-clusters and the values achieved on different clusters not connected with $U$ can take any value in $\{1, \ldots, q\}$. Hence, for the diluted Potts model, the combinatorial term appearing in expression (15) reduces to

$$
|\{\sigma: \sigma \succeq \omega, \sigma \stackrel{U}{=} 0\}|=q^{\text {number of } \omega \text {-clusters }} .
$$

In contrast, for the clock model, the larger range of edge-configurations in $\mathcal{I}^{\mathcal{E}}$ gives rise to a more delicate combinatorial structure which will be managed using the inequality (22).

\section{Proofs}

Proof of Theorem 1.1: Phase co-existence. Let us identify a disorder $J$ defined in (1) with its associated set of open edges

$$
\left\{\langle x y\rangle \in \mathcal{E}\left(\mathbb{Z}^{d}\right): J_{\langle x y\rangle}=1\right\} .
$$

We say that $C \subset \mathbb{Z}^{d}$ is a $J$-open cluster if it is a maximal set with the property that $x \stackrel{J}{\longleftrightarrow} y$ for all $x, y \in C$. Denote $x \stackrel{J}{\longleftrightarrow} \infty$ when $x$ belongs to an infinite $J$-open cluster. Let $p_{c}$ be the critical value for bond percolation in $\mathbb{Z}^{d}$. If $p>p_{c}$ then $P_{p}(J: x \stackrel{J}{\longleftrightarrow} \infty)>0$; see [GHM01, Gri06] and references therein for a treatment of percolation theory.

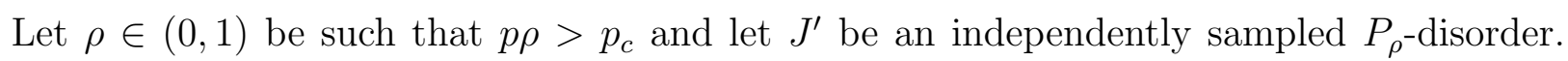
Denote by $J J^{\prime}$ the set of vertices that are open for both $J$ and $J^{\prime}$, note that $J J^{\prime}$ is a $P_{p \rho^{-}}$ disorder. Also, once $J$ is fixed, $J J^{\prime}$ is a random thinning, each open edge of $J$ is kept open with probability $\rho$ and closed with probability $(1-\rho)$, independently.

Let $\mathcal{X} \subset\{0,1\}^{\mathcal{E}\left(\mathbb{Z}^{d}\right)}$ be the set of disorders $J$ such that there is an infinite $J J^{\prime}$-open cluster with probability 1 :

$$
\mathcal{X}:=\left\{J: P_{\rho}\left(J^{\prime}: \text { there is an infinite } J J^{\prime} \text {-open cluster }\right)=1\right\} .
$$


From Fubini's Theorem, the fact that $J J^{\prime}$ is a $P_{p \rho}$-disorder, and $p \rho>p_{c}$, it is easy to see that $P_{p}(\mathcal{X})=1$. Also,

$$
\left\{J^{\prime}: \text { there is an infinite } J J^{\prime} \text {-open cluster }\right\}=\bigcup_{x \in \mathbb{Z}^{d}}\left\{J^{\prime}: x \stackrel{J J^{\prime}}{\longleftrightarrow} \infty\right\} .
$$

Hence, for each $J \in \mathcal{X}$, there exists a vertex $x \in \mathbb{Z}^{d}$ belonging to an infinite $J J^{\prime}$-open cluster with positive $P_{\rho}$-probability:

$$
P_{\rho}\left(J^{\prime}: x \stackrel{J J^{\prime}}{\longleftrightarrow} \infty\right)>0 .
$$

Let $\beta_{0}=\beta_{0}(\rho)$ be as in the statement of Theorem 2.3. Fix a disorder $J \in \mathcal{X}$ and a vertex $x$ satisfying (27). Given $n \in \mathbb{N}$, let $\Lambda_{n}:=[-n, n]^{d} \cap \mathbb{Z}^{d}$ and consider the choices

$$
V=\Lambda_{n} \cup \partial \Lambda_{n}, \quad \mathcal{E}=\left\{\langle x y\rangle \in \mathcal{E}\left(\mathbb{Z}^{d}\right):\{x, y\} \cap \Lambda_{n} \neq \emptyset, J_{\langle x y\rangle}=1\right\}, \quad U=\partial \Lambda_{n},
$$

for the vertex, edge and boundary sets in Section 2. Let $\mu, \phi$ and $B_{\rho}$ denote the clock probability on $S^{V}$, random-cluster distribution on $\mathcal{I}^{\mathcal{E}}$ and product Bernoulli probability on $\{0,1\}^{\mathcal{E}}$ associated to this choice, respectively. Note that $\mu=\mu_{\Lambda_{n}, J}^{0}$ as defined in (4) with the convention that the superscript $a$ in $\mu_{\Lambda_{n}, J}^{a}$ indicates the boundary condition $\eta_{y} \equiv a$ on $\partial \Lambda_{n}$.

Since the event $\{x \stackrel{\omega}{\longleftrightarrow} U\}$ is increasing, Theorem 2.3 implies

$$
\phi(\omega: x \stackrel{\omega}{\longleftrightarrow} U) \geq B_{\rho}(\omega: x \stackrel{\omega}{\longleftrightarrow} U)=P_{\rho}\left(J^{\prime}: x \stackrel{J J^{\prime}}{\longleftrightarrow} \partial \Lambda_{n}\right) \geq P_{\rho}\left(J^{\prime}: x \stackrel{J J^{\prime}}{\longleftrightarrow} \infty\right) .
$$

Replacing in (21) with $\mu=\mu_{\Lambda_{n}, J}^{0}$, we obtain

$$
\mu_{\Lambda_{n}, J}^{0}\left(\sigma: \sigma_{x}=0\right) \geq \mu_{\Lambda_{n}, J}^{0}\left(\sigma: \sigma_{x}=a\right)+P_{\rho}\left(J^{\prime}: x \stackrel{J J^{\prime}}{\longleftrightarrow} \infty\right), \quad \text { for any } a \neq 0 .
$$

We conclude that any weak limit $\mu_{J}^{0}$ of $\mu_{\Lambda_{n}, J}^{0}$ as $n \rightarrow \infty$ will satisfy

$$
\mu_{J}^{0}\left(\sigma: \sigma_{x}=0\right)>\mu_{J}^{0}\left(\sigma: \sigma_{x}=a\right) \quad \text { for any } a \neq 0 .
$$

By the rotational symmetry in the set $S$ of spins, the same holds with any boundary condition $b$ : the weak limit $\mu_{J}^{b}$ assigns maximal probability to having a spin $b$ at $x, \mu_{J}^{b}\left(\sigma: \sigma_{x}=b\right)>$ $\mu_{J}^{b}\left(\sigma: \sigma_{x}=a\right), a \neq b$, and therefore the $q$-Gibbs measures $\mu_{J}^{b}, b \in S$, must be different.

Proof of Proposition 2.2: Positive correlations. For any spin $a \neq 0$, by (19) and the fact that $x \stackrel{\omega}{\longleftrightarrow} U$ implies $\sigma(x)=0$,

$$
\begin{aligned}
\mu\left(\sigma: \sigma_{x}=a\right)= & \sum_{\substack{\omega \\
\omega: x \stackrel{\leftrightarrow}{\leftrightarrow} U}} \frac{\hat{\mu}\left(\sigma: \sigma_{x}=a, \omega \preceq \sigma\right)}{\hat{\mu}(\sigma: \omega \preceq \sigma)} \phi(\omega) \\
\leq & \sum_{\substack{\omega \\
\omega: x \stackrel{\leftrightarrow}{\leftrightarrow} U}} \frac{\hat{\mu}\left(\sigma: \sigma_{x}=0, \omega \preceq \sigma\right)}{\hat{\mu}(\sigma: \omega \preceq \sigma)} \phi(\omega)=Q\left((\omega, \sigma): \sigma_{x}=0, x \stackrel{\omega}{\hookrightarrow} U\right),
\end{aligned}
$$

where the inequality holds by (23). Apply (20) to conclude. 
Proof of Theorem 2.3: Stochastic domination. The measure $\phi$ gives positive probability to every edge configuration. Under this hypothesis, Holley's inequality (Theorem 4.8 of [GHM01] for instance), asserts that the stochastic domination $B_{\rho} \leq_{s t} \phi$ follows from the single-bond inequalities

$$
\rho \leq \phi\left(\omega: \omega_{\langle x y\rangle}=1 \mid \omega: \omega \stackrel{\mathcal{E} \backslash\langle x y\rangle}{=} \omega^{\prime}\right)=: \alpha\left(\langle x y\rangle, \omega^{\prime}\right), \quad\langle x y\rangle \in \mathcal{E}, \omega^{\prime} \in \mathcal{I}^{\mathcal{E}} .
$$

Given $t \in \mathcal{I}$, we define $t_{\langle x y\rangle} \omega^{\prime} \in \mathcal{I}^{\mathcal{E}}$ by

$$
\left(t_{\langle x y\rangle} \omega^{\prime}\right)_{\langle x y\rangle}=t \quad \text { and } \quad t_{\langle x y\rangle} \omega^{\prime} \stackrel{\mathcal{E} \backslash\langle x y\rangle}{=} \omega^{\prime} .
$$

Omitting the dependence of $\alpha$ on $\left(\langle x y\rangle, \omega^{\prime}\right)$ in the notation,

$$
\alpha=\frac{\phi\left(1_{\langle x y\rangle} \omega^{\prime}\right)}{\sum_{i=0}^{k} \phi\left(\left(t_{i}\right)_{\langle x y\rangle} \omega^{\prime}\right)}=\frac{r_{k}\left|\left\{\sigma: \sigma \succeq 1_{\langle x y\rangle} \omega^{\prime}, \sigma \stackrel{U}{=} 0\right\}\right|}{\sum_{i=0}^{k} r_{i}\left|\left\{\sigma: \sigma \succeq\left(t_{i}\right)_{\langle x y\rangle} \omega^{\prime}, \sigma \stackrel{U}{=} 0\right\}\right|},
$$

and

$$
\alpha^{-1}=\sum_{i=0}^{k} \frac{r_{i}}{r_{k}} \frac{\left|\left\{\sigma: \sigma \succeq\left(t_{i}\right)_{\langle x y\rangle} \omega^{\prime}, \sigma \stackrel{U}{=} 0\right\}\right|}{\left|\left\{\sigma: \sigma \succeq 1_{\langle x y\rangle} \omega^{\prime}, \sigma \stackrel{U}{=} 0\right\}\right|} .
$$

Let $(V, \tilde{\mathcal{E}})$ be the auxiliary graph obtained from $(V, \mathcal{E})$ by adding all edges connecting vertices in $U$ :

$$
\tilde{\mathcal{E}}:=\mathcal{E} \cup\{\langle u v\rangle:\{u, v\} \subset U\} .
$$

Let $\tilde{\omega} \in \mathcal{I}^{\tilde{\mathcal{E}}}$ be defined by

$$
\tilde{\omega} \stackrel{\tilde{\mathcal{E}} \backslash \mathcal{E}}{=} 1 \quad \text { and } \quad \tilde{\omega} \stackrel{\mathcal{E}}{=} \omega^{\prime} .
$$

Extend the definition of $t_{\langle x y\rangle} \tilde{\omega} \in \mathcal{I}^{\tilde{\mathcal{E}}}$ and the compatibility notion $\sigma \preceq \tilde{\omega}$ to the enlarged graph in the obvious way and use the rotation invariance of $S$ to get

$$
\left|\left\{\sigma: \sigma \succeq\left(t_{i}\right)_{\langle x y\rangle} \omega^{\prime}, \sigma \stackrel{U}{=} 0\right\}\right|=\frac{1}{q}\left|\left\{\sigma: \sigma \succeq\left(t_{i}\right)_{\langle x y\rangle} \tilde{\omega}\right\}\right|,
$$

and replacing in (33),

$$
\alpha^{-1}=\sum_{i=0}^{k} \frac{r_{i}}{r_{k}} \frac{\left|\left\{\sigma: \sigma \succeq\left(t_{i}\right)_{\langle x y\rangle} \tilde{\omega}\right\}\right|}{\left|\left\{\sigma: \sigma \succeq 1_{\langle x y\rangle} \tilde{\omega}\right\}\right|} .
$$

For $0 \leq i \leq k$, let

$$
K_{i}:=\left|\left\{(a, b) \in S \times S: W(a-b)=t_{i}\right\}\right| .
$$

We have

$$
\begin{aligned}
\left|\left\{\sigma: \sigma \succeq\left(t_{i}\right)_{\langle x y\rangle} \tilde{\omega}\right\}\right| & =\sum_{j=i}^{k}\left|\left\{\sigma: \sigma \succeq \tilde{\omega}, W\left(\sigma_{y}-\sigma_{x}\right)=t_{j}\right\}\right| \\
& =\sum_{j=i}^{k} K_{j}\left|\left\{\sigma: \sigma \succeq \tilde{\omega}, \sigma_{y}=0, \sigma_{x}=a_{j}\right\}\right|,
\end{aligned}
$$




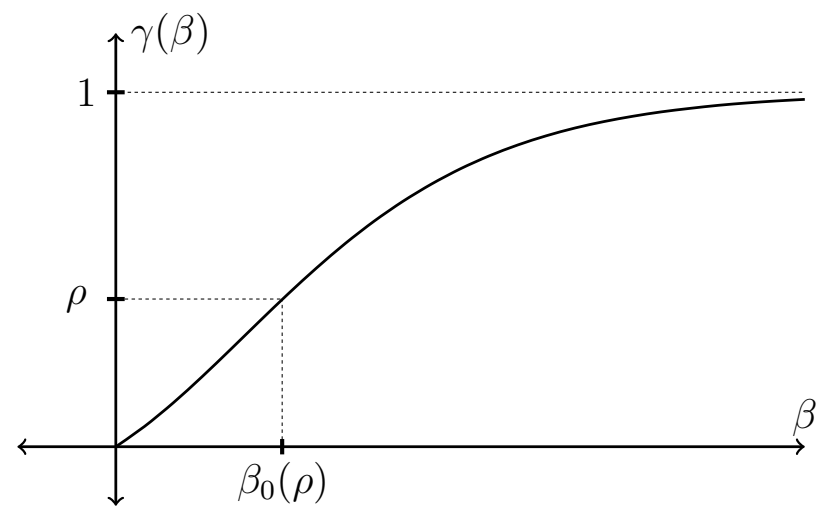

Figure 1

where $a_{j} \in S$ is an angle such that $W\left(a_{j}\right)=t_{j}$. The second identity holds again by rotation invariance. Replacing in expression (36),

$$
\alpha^{-1}=\sum_{i=0}^{k} \sum_{j=i}^{k} \frac{r_{i}}{r_{k}} \frac{K_{j}}{K_{k}} \frac{\left|\left\{\sigma: \sigma \succeq \tilde{\omega}, \sigma_{y}=0, \sigma_{x}=a_{j}\right\}\right|}{\left|\left\{\sigma: \sigma \succeq \tilde{\omega}, \sigma_{y}=0, \sigma_{x}=0\right\}\right|} .
$$

By Lemma 2.4 applied to $U=\{y\}$ we get

$$
\alpha^{-1} \leq \sum_{i=0}^{k} \sum_{j=i}^{k} \frac{r_{i}}{r_{k}} \frac{K_{j}}{K_{k}}=\sum_{j=0}^{k} \frac{t_{j}}{r_{k}} \frac{K_{j}}{K_{k}}
$$

since $t_{j}=\sum_{i=0}^{j} r_{i}$. From (31), we conclude that the stochastic domination $B_{\rho} \leq_{s t} \phi$ will follow for $\beta$ satisfying

$$
\rho \leq \gamma(\beta):=\left(\sum_{j=0}^{k} \frac{t_{j}}{r_{k}} \frac{K_{j}}{K_{k}}\right)^{-1} .
$$

The function $\gamma$ is increasing. Indeed, for each $j, \frac{r_{k}}{t_{j}}$ is of the form $e^{\beta A}\left(1-e^{-\beta B}\right)$ with $A$ and $B$ positive numbers, and hence increasing. On the other hand $\lim _{\beta \rightarrow \infty} r_{k}=1$ and $\lim _{\beta \rightarrow \infty} t_{i}=0$ for $i<k$; as a consequence, $\lim _{\beta \rightarrow \infty} \gamma(\beta)=1$. Finally, $\lim _{\beta \downarrow 0} r_{k}=0$ and $\lim _{\beta \downarrow 0} t_{i}=1$ for every $i$, so $\lim _{\beta \downarrow 0} \gamma(\beta)=0$. See Figure 1 for the graph of $\gamma$ when $q=4$. In particular, $\gamma$ is injective and its inverse $\gamma^{-1}:(0,1) \rightarrow(0, \infty)$ is well defined. We conclude that if $\beta_{0}=\gamma^{-1}(\rho)$, then equation (31) holds for $\beta \geq \beta_{0}$.

Proof of Lemma 2.4. The case $x \in U$ is trivial, so let us suppose $x \in V \backslash U$. If $|U|>1$ the model can be reduced to the case $|U|=1$ by identifying all vertices in $U$. We may then suppose $U=\{y\}$ for some $y \neq x$.

Let

$$
L_{\omega}(a):=\left\{\sigma: \sigma \succeq \omega, \sigma_{y}=0, \sigma_{x}=a\right\} .
$$

We will construct an injection $F: L_{\omega}(a) \hookrightarrow L_{\omega}(0)$. Here is a brief description of the procedure. Fix $a \in S$ and consider the reflection $R: S \rightarrow S$ with respect to the line $\ell$ at angle $a / 2$ with the horizontal axis (see Figure 2), that is, $R b=a-b \bmod 2 \pi$. Clearly, $R(a)=0$. We progressively transform an initial configuration $\sigma \in L_{\omega}(a)$ into a configuration $\sigma^{\prime} \in L_{\omega}(0)$. The first step is to modify $\sigma$ by applying the reflection $R$ to the spin at the vertex $x$. The resulting 


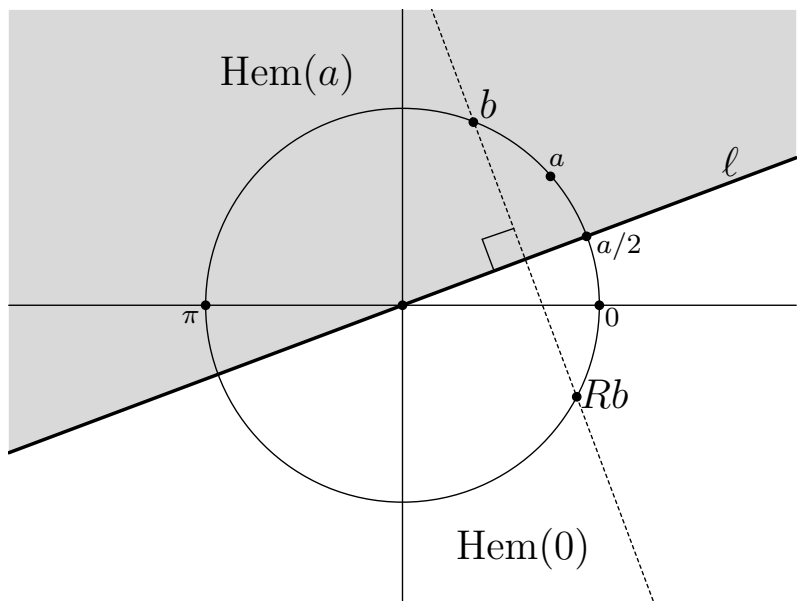

Figure 2

configuration may present incompatibilities with respect to $\omega$ and, if it does, they will appear at edges $\{\langle u x\rangle\}_{u \in V}$. If this is the case, we modify the configuration by applying the transformation $R$ to the spins of the conflicting vertices. We obtain a configuration without incompatibilities in the edges having one endpoint at $x$, but we might have created new incompatibilities at a second level of edges, that is, edges with one endpoint at a vertex that is a neighbour of $x$. We solve this by applying $R$ once more to the spins of the new conflicting vertices, and keep repeating the procedure until there are no more incompatibilities. We need to show that the resulting configuration $\sigma^{\prime}$ belongs to $L_{\omega}(0)$, and that the construction is indeed injective. The most delicate part is to prove that this process stops before reaching the vertex $y$.

It suffices to prove the result when $a \neq 0$, which we assume from now on. We may also assume that $a \in(0, \pi]$, as the other case is symmetric. As before, the boundary $\partial V^{\prime}$ of a vertex set $V^{\prime} \subset V$ denotes the set of vertices $u \in V \backslash V^{\prime}$ such that $\langle u v\rangle \in \mathcal{E}$ for some $v \in V^{\prime}$.

Let now $\sigma \in L_{\omega}(a)$. Define a sequence of sets $A_{0} \subset A_{1} \subset \cdots \subset V$ associated to $\sigma$ by $A_{0}:=\{x\}$ and, for $n \geq 0$,

$$
A_{n+1}:=A_{n} \cup\left\{u \in \partial A_{n}: W\left(\sigma_{u}-R \sigma_{v}\right)<\omega_{\langle u v\rangle} \text { for some } v \in A_{n}\right\} .
$$

At each step, $A_{n+1} \backslash A_{n}$ consists of those vertices where new incompatibilities would arise when applying the reflection to $A_{n}$. Let

$$
A:=\bigcup_{n \geq 0} A_{n}
$$

Define the function $F: L_{\omega}(a) \rightarrow S^{V}$ by

$$
(F \sigma)_{u}:= \begin{cases}R \sigma_{u} & \text { if } u \in A \\ \sigma_{u} & \text { if } u \notin A .\end{cases}
$$

We now show that $i)$ the image of $F$ is contained in $L_{\omega}(0)$ and $\left.i i\right)$ that $F: L_{\omega}(a) \rightarrow L_{\omega}(0)$ is an injection.

i) $F \sigma \in L_{\omega}(0)$. In order to prove that $F \sigma \succeq \omega$, we need to show that

$$
W\left((F \sigma)_{u}-(F \sigma)_{v}\right) \geq \omega_{\langle u v\rangle}
$$

for any $\langle u v\rangle \in \mathcal{E}$. The cases $\{u, v\} \subset A$ or $\{u, v\} \subset A^{c}$ are trivial. If $u \notin A$ and $v \in A$, condition (47) reads $W\left(\sigma_{u}-R \sigma_{v}\right) \geq \omega_{\langle u v\rangle}$, which must hold; otherwise $u$ would have belonged to $A$ in the first place. 
It remains to prove that $(F \sigma)_{y}=0$, which follows if we show that $y \notin A$. The line $\ell$ (see Figure 2) separates the two open hemispheres $\operatorname{Hem}(0)$ and $\operatorname{Hem}(a)$ defined by

$$
\begin{aligned}
& \operatorname{Hem}(0):=\{b \in S: \sin (b-a / 2)<0\} \\
& \operatorname{Hem}(a):=\{b \in S: \sin (b-a / 2)>0\} .
\end{aligned}
$$

Since $0 \in \operatorname{Hem}(0)$, it is enough to prove that $\sigma_{u} \in \operatorname{Hem}(a)$ for every $u \in A \backslash\{x\}$. We proceed by induction. If $A_{1} \neq \emptyset$, let $u \in A_{1} \backslash\{x\}$ with $\sigma_{u}=b$. By the definition of $A_{1}$, we have $W(b-0)<\omega_{\langle u x\rangle} \leq W(b-a)$, where the inequality follows from the fact that $\sigma \succeq \omega$. Now, $W(b-0)<W(b-a)$ is equivalent to $\cos (b)<\cos (b-a)$. But

$$
\begin{gathered}
\cos (b)<\cos (b-a) \quad \Longleftrightarrow \cos \left(b-\frac{a}{2}+\frac{a}{2}\right)<\cos \left(b-\frac{a}{2}-\frac{a}{2}\right) \\
\Longleftrightarrow \cos \left(b-\frac{a}{2}\right) \cos \left(\frac{a}{2}\right)-\sin \left(b-\frac{a}{2}\right) \sin \left(\frac{a}{2}\right) \\
<\cos \left(b-\frac{a}{2}\right) \cos \left(\frac{a}{2}\right)+\sin \left(b-\frac{a}{2}\right) \sin \left(\frac{a}{2}\right) \\
\Longleftrightarrow \quad 0<2 \sin \left(b-\frac{a}{2}\right) \sin \left(\frac{a}{2}\right) \quad \Longleftrightarrow \quad 0<\sin \left(b-\frac{a}{2}\right) \quad \Longleftrightarrow b \in \operatorname{Hem}(a),
\end{gathered}
$$

and the claim holds for $A_{1}$. Suppose now that $\sigma_{u} \in \operatorname{Hem}(a)$, that is

$$
\sin \left(\sigma_{u}-\frac{a}{2}\right)>0
$$

for every $u \in A_{n}$. If $A_{n+1} \neq \emptyset$, let $v \in A_{n+1}$ and $w \in A_{n}$ be such that $W\left(\sigma_{v}-R \sigma_{w}\right)<$ $W\left(\sigma_{v}-\sigma_{w}\right)$, which is equivalent to $\cos \left(\sigma_{v}-\left(a-\sigma_{w}\right)\right)<\cos \left(\sigma_{v}-\sigma_{w}\right)$. By the inductive hypothesis $\sin \left(\sigma_{w}-\frac{a}{2}\right)>0$. An argument similar to the one leading from (48) to (50) yields

$$
0<2 \sin \left(\sigma_{v}-\frac{a}{2}\right) \sin \left(\sigma_{w}-\frac{a}{2}\right)
$$

and then $0<\sin \left(\sigma_{v}-\frac{a}{2}\right)$, i.e. $\sigma_{v} \in \operatorname{Hem}(a)$. This completes the induction.

ii) $F$ is injective. Let $\sigma, \sigma^{\prime} \in L_{\omega}(0, a)$ be two different configurations and denote by $A, A_{1}, A_{2}, \ldots$ and $A^{\prime}, A_{1}^{\prime}, A_{2}^{\prime}, \ldots$ their associated incompatibility sets. If $A=A^{\prime}$, we are done because $R$ is injective. Suppose $A \neq A^{\prime}$ and let

$$
n=\min \left\{j \geq 1: A_{j} \neq A_{j}^{\prime}\right\} ;
$$

so that in particular $A_{n-1}=A_{n-1}^{\prime}$. If there is a vertex $u \in A_{n-1}$ such that $\sigma_{u} \neq \sigma_{u}^{\prime}$, we are done. Suppose $\sigma \stackrel{A_{n-1}}{=} \sigma^{\prime}$. Without loss of generality, let us take $u \in A_{n} \backslash A_{n}^{\prime}$. We claim that $(F \sigma)_{u} \neq\left(F \sigma^{\prime}\right)_{u}$. We know that $\sigma_{u} \neq \sigma_{u}^{\prime}$, as otherwise we would have $u \in A_{n}^{\prime}$. If $u \in A^{\prime}$ we are done. Suppose then $u \notin A^{\prime}$. Let $v \in A_{n-1}$ be such that $W\left(\sigma_{u}-R \sigma_{v}\right)<\omega_{\langle u v\rangle}$. Using that $W\left(a^{\prime}-b^{\prime}\right)=W\left(R a^{\prime}-R b^{\prime}\right)$ for any $a^{\prime}, b^{\prime} \in S$, that $R^{2}$ is the identity and that $(F \sigma)_{u}=R \sigma_{u}$, we have $W\left(\sigma_{u}-R \sigma_{v}\right)=W\left((F \sigma)_{u}-\sigma_{v}\right)$, and then

$$
W\left((F \sigma)_{u}-\sigma_{v}\right)<\omega_{\langle u v\rangle} .
$$

On the other hand, since $\sigma^{\prime} \succeq \omega$, we have $W\left(\sigma_{u}^{\prime}-\sigma_{v}^{\prime}\right) \geq \omega_{\langle u v\rangle}$. But $W\left(\sigma_{u}^{\prime}-\sigma_{v}^{\prime}\right)=W\left(\left(F \sigma^{\prime}\right)_{u}-\sigma_{v}\right)$ because $u \notin A^{\prime}$ and $\sigma \stackrel{A_{n-1}}{=} \sigma^{\prime}$, and hence

$$
W\left(\left(F \sigma^{\prime}\right)_{u}-\sigma_{v}\right) \geq \omega_{\langle u v\rangle} .
$$

From inequalities (52) and (53) we obtain $(F \sigma)_{u} \neq\left(F \sigma^{\prime}\right)_{u}$, as claimed. 


\section{Appendix}

Proof of Theorem 2.1: The Edwards-Sokal random-cluster representation. The first step is to write the density of $\mu$ with respect to $\hat{\mu}$ :

$$
\mu(\sigma)=\hat{\mu}(\sigma) \frac{1}{Z^{\prime}} \prod_{\langle x y\rangle \in \mathcal{E}} W\left(\sigma_{x}-\sigma_{y}\right),
$$

with $Z^{\prime}$ the normalizing constant in (17). Since $W\left(\sigma_{x}-\sigma_{y}\right)=\theta\left(t \in \mathcal{I}: t \leq W\left(\sigma_{x}-\sigma_{y}\right)\right)$, the weight of a spin configuration can be realized as the probability of a related event on the associated edge set:

$$
\prod_{\langle x y\rangle \in \mathcal{E}} W\left(\sigma_{x}-\sigma_{y}\right)=\hat{\phi}\left(\bigcap_{\langle x y\rangle \in \mathcal{E}}\left\{\omega \in \mathcal{I}^{\mathcal{E}}: \omega_{\langle x y\rangle} \leq W\left(\sigma_{x}-\sigma_{y}\right)\right\}\right)=\hat{\phi}(\omega: \omega \preceq \sigma) .
$$

Here is where the definition of compatibility appears naturally. Inserting (55) in (54), we get

$$
\mu(\sigma)=\sum_{\omega \in \mathcal{I}^{\mathcal{E}}} \frac{1}{Z^{\prime}} \mathbf{1}[\omega \preceq \sigma] \hat{\phi}(\omega) \hat{\mu}(\sigma)=\sum_{\omega \in \mathcal{I}^{\mathcal{E}}} Q((\omega, \sigma)) .
$$

Hence, $\mu$ is the second marginal of $Q$. Adding over all the possible vertex-configurations, it is easy to see that $\phi$ is its first marginal.

Asymptotics for $\beta_{0}$. The threshold $\beta_{0}$ introduced in Theorem 1.1 is $\beta_{0}=\gamma^{-1}(\rho)$, where $\gamma:(0, \infty) \rightarrow(0,1)$ is the function defined in the proof of Theorem 2.3, and $\rho$ is the parameter defined in the proof of Theorem 1.1, such that $\rho>\frac{p_{c}}{p}$. Since $\gamma^{-1}$ is increasing, we can take the infimum over $\rho$ to optimize $\beta_{0}=\gamma^{-1}\left(\frac{p_{c}}{p}\right)$.

For any fixed $\beta>0$ we have that $\lim _{q \rightarrow \infty} \gamma(\beta)=0$. Indeed, $\lim _{q \rightarrow \infty} r_{k}=0$ and $t_{i} \frac{K_{i}}{K_{k}}$ is bounded away from zero uniformly in $q$. As a consequence, for every fixed $\tilde{p} \in(0,1)$, $\lim _{q \rightarrow \infty} \gamma^{-1}(\tilde{p})=\infty$. We conclude that our method is not informative as a discretization of the $X Y$ model, that is, when the number of spins goes to infinity.

Note that

$$
\gamma(\beta)^{-1}=\frac{1}{r_{k}}+\sum_{i=0}^{k-1} \frac{t_{i}}{r_{k}} \frac{K_{i}}{K_{k}} \leq \frac{1}{r_{k}}+\sum_{i=0}^{k-1} \frac{t_{k-1}}{r_{k}} 2 \leq \frac{1}{r_{k}}+\frac{t_{k-1}}{r_{k}} q,
$$

so that

$$
\gamma(\beta) \geq \frac{r_{k}}{1+q t_{k-1}} .
$$

Then $\beta_{0}$ is bounded above by the solution to the equation

$$
\frac{p_{c}}{p}=\frac{r_{k}}{1+q t_{k-1}} \text {. }
$$

Using that $r_{k}=1-t_{k-1}$ and that $t_{k-1}=e^{-\beta\left(1-\cos \left(\frac{2 \pi}{q}\right)\right)}$, this solution can be explicitly computed as

$$
\frac{\log \left(\frac{p+q p_{c}}{p-p_{c}}\right)}{1-\cos \left(\frac{2 \pi}{q}\right)}
$$


If we fix $p$ and $d$, this expression is of order $q^{2} \log (q)$ as $q \rightarrow \infty$, the same order given by Pirogov-Sinai theory and reflection positivity in the 2-dimensional homogeneous case. If we fix $p$ and $q$, it is of order

$$
\frac{\log \left(1+\frac{1}{d}\right)}{1-\cos \left(\frac{2 \pi}{q}\right)}
$$

as $d \rightarrow \infty$, taking into account that $p_{c} \sim \frac{1}{2 d}$. In particular, $\beta_{0} \rightarrow 0$ as $d \rightarrow \infty$.

\section{Acknowledgments}

We are grateful to both referees for several comments that helped us give a clear focus to the article. Nahuel Soprano-Loto would like to thank Roberto Fernández for many useful discussions and his warm welcome to the University of Utrecht, a visit funded by grant 2010-MINCYT-NIO Interacting stochastic systems: fluctuations, hydrodynamics, scaling limits. This research has been supported by grant PICT 2012-2744 Stochastic Processes and Statistical Mechanics.

\section{References}

[ACCN87] M. Aizenman, J. T. Chayes, L. Chayes, and C. M. Newman. The phase boundary in dilute and random Ising and Potts ferromagnets. J. Phys. A, 20(5):L313-L318, 1987.

[Bis09] Marek Biskup. Reflection positivity and phase transitions in lattice spin models. In Methods of contemporary mathematical statistical physics, volume 1970 of Lecture Notes in Math., pages 1-86. Springer, Berlin, 2009.

[Dob68] R. L. Dobrushin. The description of a random field by means of conditional probabilities and conditions of its regularity. Theor. Prob. Appl., 13:197-224, 1968.

[Dub11] Julien Dubédat. Topics on abelian spin models and related problems. Probab. Surv., 8:374-402, 2011.

[ES88] Robert G. Edwards and Alan D. Sokal. Generalization of the Fortuin-KasteleynSwendsen-Wang representation and Monte Carlo algorithm. Phys. Rev. D (3), 38(6):2009-2012, 1988.

[FILS78] Jürg Fröhlich, Robert Israel, Elliott H. Lieb, and Barry Simon. Phase transitions and reflection positivity. I. General theory and long range lattice models. Comm. Math. Phys., 62(1):1-34, 1978.

[FSL] Roberto Fernández and Nahuel Soprano-Loto. In preparation.

[GHM01] Hans-Otto Georgii, Olle Häggström, and Christian Maes. The random geometry of equilibrium phases. In Phase transitions and critical phenomena, Vol. 18, volume 18 of Phase Transit. Crit. Phenom., pages 1-142. Academic Press, San Diego, CA, 2001. 
[Gri06] Geoffrey Grimmett. The random-cluster model, volume 333 of Grundlehren der Mathematischen Wissenschaften [Fundamental Principles of Mathematical Sciences]. Springer-Verlag, Berlin, 2006.

[KF69] P. W. Kasteleyn and C. M. Fortuin. Phase transitions in lattice systems with random local properties. Journal of the Physical Society of Japan, 26:11-14, 1969.

[MS11] Christian Maes and Senya Shlosman. Rotating states in driven clock- and XYmodels. J. Stat. Phys., 144(6):1238-1246, 2011.

[PS75] S. A. Pirogov and Ja. G. Sinal. Phase diagrams of classical lattice systems. Teoret. Mat. Fiz., 25(3):358-369, 1975.

[vdBM94] J. van den Berg and C. Maes. Disagreement percolation in the study of Markov fields. Ann. Probab., 22(2):749-763, 1994.

[vEKO11] Aernout C. D. van Enter, Christof Külske, and Alex A. Opoku. Discrete approximations to vector spin models. J. Phys. A, 44(47):475002, 11, 2011. 